Appendix I: ${ }^{40} \mathrm{Ar} /{ }^{39} \mathrm{Ar}$ and $\mathrm{K}$-Ar Isotopic Data:

for the paper by John H. Dilles and Phillip B. Gans, 1994, The chronology of Cenozoic volcanism and deformation in the Yerington area, western Basin-and-Range and Walker Lane

\title{
A. ${ }^{40} \mathrm{Ar} /{ }^{39} \mathrm{Ar}$ step-heating isotopic data.
}

For the fifteen samples listed below, temperature is given in degrees Centigrade $\left(\mathrm{T}^{\circ} \mathrm{C}\right)$. $40(v)=$ volts corrected for blank and reactor-produced $40 \mathrm{Ar}$; $40(\mathrm{~mol}) \sim 40(\mathrm{v}) \cdot 2.3367 \cdot 10^{-14}$, where $40(\mathrm{~mol})=$ moles of $40 \mathrm{Ar}$ corrected for blank and reactor-produced $40 \mathrm{Ar}$.

$\mathrm{K} / \mathrm{Ca}$ is base-10 logarithm, $\sum 39 \mathrm{Ar}$ is cumulative, $40 \mathrm{Ar}^{*}=$ radiogenic fraction.

Note, for small amounts of radiogenic $40 \mathrm{Ar}$, subtraction of blanks and backgrounds, with included errors, may yield a $40 \mathrm{Ar}$ radiogenic fraction listed in table as a negative value; these negative values should be treated as near-zero radiogenic $40 \mathrm{Ar}$ fractions.

Sample 1: cvi122 YR240B Hb J=0.0055670

$\begin{array}{llllllllll}\mathrm{T} & 40(\mathrm{v}) & 40 / 39 & 37 / 39 & 36 / 39 & \mathrm{~K} / \mathrm{Ca} & \sum 39 \mathrm{Ar} & 40 \mathrm{Ar} * & \text { Age }(\mathrm{Ma}) & \pm \\ 700 & 3.8479 & -9.6707 & 3.7409 & 1.6928 & -0.88 & 0.002 & -0.020 & -99.84 & 211.55 \\ 850 & 0.1764 & 2.1214 & 5.0924 & 0.1069 & -1.02 & 0.004 & 0.052 & 21.18 & 15.53 \\ 980 & 0.6825 & 0.6321 & 6.5326 & 0.0081 & -1.13 & 0.090 & 0.048 & 6.34 & 0.74 \\ 1025 & 1.0743 & 0.6130 & 6.5547 & 0.0027 & -1.13 & 0.462 & 0.108 & 6.15 & 0.27 \\ 1050 & 0.9421 & 0.6145 & 6.5865 & 0.0025 & -1.13 & 0.821 & 0.117 & 6.16 & 0.26 \\ 1100 & 0.3578 & 0.6163 & 6.9981 & 0.0028 & -1.16 & 0.946 & 0.073 & 6.18 & 0.29 \\ 1200 & 0.1660 & 0.6230 & 7.4307 & 0.0065 & -1.18 & 0.973 & 0.020 & 6.25 & 1.33 \\ 1400 & 0.1607 & 0.7916 & 8.3207 & 0.0058 & -1.23 & 1.000 & 0.073 & 7.93 & 1.00\end{array}$

Sample 2: YR-1 Plag SB2A-52

$\begin{array}{llllllllll}\mathrm{T} & 40(\mathrm{v}) & 40 / 39 & 37 / 39 & 36 / 39 & \mathrm{~K} / \mathrm{Ca} & \sum 39 \mathrm{Ar} & 40 \mathrm{Ar} * & \text { Age(Ma) } & \pm \\ 550 & 0.4604 & 2.359 & 34.8533 & 0.0272 & -1.85 & 0.154 & 0.303 & 7.45 & 0.43 \\ 625 & 0.37 & 2.3415 & 37.2001 & 0.0192 & -1.89 & 0.339 & 0.45 & 7.39 & 0.32 \\ 700 & 0.4358 & 2.4407 & 38.0009 & 0.0179 & -1.89 & 0.575 & 0.505 & 7.71 & 0.31 \\ 725 & 0.3406 & 2.3238 & 38.6831 & 0.0185 & -1.92 & 0.758 & 0.48 & 7.34 & 0.36 \\ 850 & 0.1946 & 2.5977 & 38.4795 & 0.0206 & -1.92 & 0.847 & 0.451 & 8.2 & 0.58 \\ 925 & 0.1216 & 2.6848 & 33.7526 & 0.033 & -1.85 & 0.879 & 0.269 & 8.48 & 2.19 \\ 1000 & 0.3755 & 1.2328 & 28.8091 & 0.0933 & -1.77 & 0.915 & 0.046 & 3.9 & 1.76 \\ 1100 & 0.297 & 2.4057 & 35.1117 & 0.061 & -1.85 & 0.957 & 0.133 & 7.6 & 1.3 \\ 1250 & 0.262 & 3.3874 & 32.2789 & 0.0502 & -1.82 & 1 & 0.212 & 10.69 & 1.82\end{array}$

\section{$\mathrm{J}=0.0017540$}

Total fusion age, TFA $=7.59 \pm 0.20 \mathrm{Ma}$ (including $\mathrm{J}$ )

Weighted mean plateau age, WMPA $=7.54 \pm 0.17 \mathrm{Ma}$ (including $\mathrm{J}$ )

Inverse isochron age $=7.53 \pm 0.33 \mathrm{Ma}$. (MSWD $=0.44 ; 40 \mathrm{Ar} / 36 \mathrm{Ar}=296.1 \pm 5.3$ )

Steps used: $550,625,700,775,850,925,1100$, 
Sample 3: 93-87-S6 plag (cvi125) $\quad \mathrm{J}=0.0055400$

$\begin{array}{llllllllll}\mathrm{T} & 40(v) & 40 / 39 & 37 / 39 & 36 / 39 & \mathrm{~K} / \mathrm{Ca} & \sum 39 \mathrm{Ar} & 40 \mathrm{Ar} * & \text { Age(Ma) } & \pm \\ 600 & 0.2523 & 0.9128 & 7.7873 & 0.0482 & -1.20 & 0.037 & 0.063 & 9.1 & 4.64 \\ 700 & 2.4481 & 4.1263 & 12.4348 & 0.2528 & -1.41 & 0.103 & 0.053 & 40.77 & 25.24 \\ 800 & 0.2685 & 0.9228 & 15.432 & 0.017 & -1.51 & 0.222 & 0.193 & 9.2 & 1.56 \\ 900 & 0.2666 & 0.9128 & 17.0923 & 0.0173 & -1.55 & 0.341 & 0.194 & 9.1 & 1.98 \\ 1000 & 0.3339 & 0.8846 & 17.8238 & 0.0208 & -1.57 & 0.465 & 0.156 & 8.82 & 3.06 \\ 1100 & 0.3265 & 0.8746 & 18.0868 & 0.0178 & -1.57 & 0.61 & 0.185 & 8.72 & 2.29 \\ 1200 & 0.3373 & 1.0299 & 18.1232 & 0.019 & -1.57 & 0.745 & 0.196 & 10.26 & 1.81 \\ 1300 & 0.2493 & 1.0179 & 18.7452 & 0.0214 & -1.59 & 0.834 & 0.173 & 10.14 & 3.38 \\ 1400 & 0.369 & 0.8657 & 18.7214 & 0.0178 & -1.59 & 1 & 0.184 & 8.63 & 1.72\end{array}$

Total fusion age, TFA $=11.30 \pm 1.86 \mathrm{Ma}$ (including $\mathrm{J}$ )

Weighted mean plateau age, WMPA $=9.23 \pm 0.76 \mathrm{Ma}$ (including $\mathrm{J}$ )

Inverse isochron age $=9.09 \pm 2.08 \mathrm{Ma}$. (MSWD $=0.10 ; 40 \mathrm{Ar} / 36 \mathrm{Ar}=296.4 \pm 4.2$ )

Steps used: $600,800,900,1000,1100,1200,1300,1400$, or $93 \% \sum 39 \mathrm{Ar}$

Sample 4: cvi118 110JD91 $\mathrm{Hb} \quad \mathrm{J}=0.0055933$

$\begin{array}{llllllllll}\mathrm{T} & 40(\mathrm{v}) & 40 / 39 & 37 / 39 & 36 / 39 & \mathrm{~K} / \mathrm{Ca} & \sum 39 \mathrm{Ar} & 40 \mathrm{Ar} * & \text { Age(Ma) } & \pm \\ 700 & 6.9463 & 0.4870 & 0.4998 & 0.2130 & -0.01 & 0.020 & 0.007 & 4.91 & 18.44 \\ 800 & 0.2723 & 0.8448 & 0.3028 & 0.0060 & 0.21 & 0.040 & 0.319 & 8.50 & 0.98 \\ 900 & 0.5773 & 0.8884 & 0.2265 & 0.0112 & 0.33 & 0.065 & 0.210 & 8.94 & 1.26 \\ 1000 & 0.8861 & 0.8402 & 1.9777 & 0.0115 & -0.61 & 0.106 & 0.168 & 8.46 & 1.26 \\ 1030 & 0.8710 & 0.8576 & 4.7018 & 0.0029 & -0.98 & 0.226 & 0.364 & 8.63 & 0.35 \\ 1060 & 2.5762 & 0.8690 & 5.2264 & 0.0017 & -1.03 & 0.734 & 0.483 & 8.75 & 0.16 \\ 1100 & 0.6492 & 0.8793 & 3.6344 & 0.0012 & -0.87 & 0.861 & 0.623 & 8.85 & 0.16 \\ 1200 & 0.7414 & 0.8875 & 2.1621 & 0.0011 & -0.65 & 0.994 & 0.695 & 8.93 & 0.11 \\ 1400 & 0.0796 & 0.9145 & 6.6623 & 0.0069 & -1.14 & 1.000 & 0.161 & 9.20 & 2.87\end{array}$

Sample 5: f104 (YR-13B) Biotite $\quad J=0.0055953$

$\begin{array}{llllllllll}\mathrm{T} & 40(\mathrm{v}) & 40 / 39 & 37 / 39 & 36 / 39 & \mathrm{~K} / \mathrm{Ca} & \sum 39 \mathrm{Ar} & 40 \mathrm{Ar} * & \text { Age(Ma) } & \pm \\ 650 & 0.3940 & 0.5775 & 0.0006 & 0.0246 & 2.91 & 0.004 & 0.075 & 5.82 & 2.20 \\ 750 & 4.9028 & 0.3250 & 0.0001 & 0.0867 & 3.83 & 0.021 & 0.013 & 3.28 & 6.99 \\ 830 & 2.3120 & 0.8046 & 0.0000 & 0.0125 & 4.39 & 0.067 & 0.181 & 8.10 & 1.03 \\ 920 & 1.5613 & 0.8940 & 0.0000 & 0.0023 & 5.78 & 0.156 & 0.576 & 9.00 & 0.16 \\ 1000 & 1.9349 & 0.8844 & 0.0000 & 0.0020 & 4.63 & 0.273 & 0.603 & 8.91 & 0.20 \\ 1050 & 2.6822 & 0.8896 & 0.0000 & 0.0025 & 5.23 & 0.419 & 0.549 & 8.96 & 0.21 \\ 1100 & 4.0840 & 0.8850 & 0.0000 & 0.0024 & 5.68 & 0.646 & 0.557 & 8.91 & 0.18 \\ 1150 & 3.6846 & 0.8828 & 0.0000 & 0.0015 & 5.25 & 0.893 & 0.668 & 8.89 & 0.13 \\ 1200 & 1.1535 & 0.8864 & 0.0000 & 0.0009 & 4.58 & 0.982 & 0.771 & 8.93 & 0.09 \\ 1300 & 0.2746 & 0.8529 & 0.0000 & 0.0017 & 4.21 & 1.000 & 0.632 & 8.59 & 0.26\end{array}$

NOTE: So much time elapsed between irradiation and analysis, that the correction for 37 decay was ignored. 
Sample 6: cvi116 74JD91 hbl $\mathrm{J}=0.0056069$

$\begin{array}{llllllllll}\mathrm{T} & 40(\mathrm{v}) & 40 / 39 & 37 / 39 & 36 / 39 & \mathrm{~K} / \mathrm{Ca} & \sum 39 \mathrm{Ar} & 40 \mathrm{Ar} * & \text { Age(Ma) } & \pm \\ 700 & 3.1875 & 0.0164 & 0.3747 & 0.1486 & 0.12 & 0.016 & -0.000 & 0.17 & 15.46 \\ 800 & 0.3736 & 1.0210 & 0.2406 & 0.0104 & 0.31 & 0.037 & 0.248 & 10.30 & 1.07 \\ 900 & 0.3934 & 1.3666 & 0.2679 & 0.0077 & 0.26 & 0.062 & 0.373 & 13.77 & 1.17 \\ 1000 & 8.8018 & 34.3972 & 2.3308 & 0.0096 & -0.68 & 0.116 & 0.923 & 318.14 & 0.88 \\ 1040 & 2.3163 & 1.4414 & 5.4362 & 0.0032 & -1.05 & 0.386 & 0.520 & 14.52 & 0.27 \\ 1060 & 2.4279 & 1.0969 & 5.5960 & 0.0018 & -1.06 & 0.854 & 0.555 & 11.06 & 0.15 \\ 1100 & 1.1599 & 2.3181 & 5.7481 & 0.0022 & -1.07 & 0.959 & 0.745 & 23.30 & 0.24 \\ 1200 & 1.4703 & 10.4306 & 11.3776 & 0.0064 & -1.37 & 0.988 & 0.833 & 102.53 & 0.85 \\ 1400 & 0.2797 & 3.3155 & 9.3638 & 0.0099 & -1.28 & 1.000 & 0.466 & 33.23 & 1.98\end{array}$

Sample 7: f102 (YR-7B) Biotite

$\begin{array}{llllllllll}\mathrm{T} & 40(\mathrm{v}) & 40 / 39 & 37 / 39 & 36 / 39 & \mathrm{~K} / \mathrm{Ca} & \sum 39 \mathrm{Ar} & 40 \mathrm{Ar} * & \text { Age(Ma) } \pm \\ 650 & 0.4109 & 0.3193 & 0.0013 & 0.0863 & 2.57 & 0.002 & 0.013 & 3.24 & 9.52 \\ 750 & 4.2116 & 0.2153 & 0.0006 & 0.3024 & 2.92 & 0.008 & 0.003 & 2.19 & 24.09 \\ 825 & 2.1345 & 0.9762 & 0.0001 & 0.0498 & 3.61 & 0.025 & 0.063 & 9.90 & 3.85 \\ 900 & 1.1530 & 1.2550 & 0.0000 & 0.0037 & 4.34 & 0.087 & 0.537 & 12.71 & 0.31 \\ 975 & 1.5846 & 1.2496 & 0.0000 & 0.0016 & 4.22 & 0.201 & 0.722 & 12.66 & 0.16 \\ 1035 & 2.7317 & 1.2543 & 0.0000 & 0.0016 & 4.71 & 0.398 & 0.723 & 12.71 & 0.12 \\ 1085 & 3.8948 & 1.2432 & 0.0000 & 0.0019 & 4.87 & 0.669 & 0.692 & 12.59 & 0.15 \\ 1125 & 2.6438 & 1.2308 & 0.0000 & 0.0023 & 4.53 & 0.843 & 0.648 & 12.47 & 0.21 \\ 1190 & 2.1981 & 1.2436 & 0.0000 & 0.0024 & 4.44 & 0.984 & 0.642 & 12.60 & 0.21 \\ 1300 & 0.3047 & 1.2453 & 0.0001 & 0.0040 & 3.57 & 1.000 & 0.514 & 12.62 & 0.50\end{array}$

NOTE: So much time elapsed between irradiation and analysis, that the correction for 37 decay was ignored.

Sample 8: cvi124 YR-7 hbl $\mathrm{J}=0.0055508$

$\begin{array}{llllllllll}\mathrm{T} & 40(\mathrm{v}) & 40 / 39 & 37 / 39 & 36 / 39 & \mathrm{~K} / \mathrm{Ca} & \sum 39 \mathrm{Ar} & 40 \mathrm{Ar} * & \text { Age(Ma) } & \pm \\ 600 & 0.1859 & -0.4440 & 2.3817 & 0.1424 & -0.69 & 0.002 & -0.015 & -4.45 & 27.29 \\ 700 & 2.8195 & -4.4069 & 4.0491 & 1.1250 & -0.92 & 0.005 & -0.014 & -44.68 & 115.52 \\ 800 & 0.2045 & 0.9675 & 2.3971 & 0.0519 & -0.69 & 0.010 & 0.049 & 9.66 & 5.96 \\ 900 & 0.1732 & 0.6776 & 5.6711 & 0.0603 & -1.06 & 0.013 & 0.013 & 6.77 & 7.89 \\ 1000 & 1.1907 & 1.1441 & 9.6468 & 0.0120 & -1.30 & 0.127 & 0.096 & 11.42 & 1.22 \\ 1060 & 3.8267 & 1.2215 & 9.4523 & 0.0054 & -1.29 & 0.827 & 0.229 & 12.19 & 0.53 \\ 1110 & 0.6516 & 1.2194 & 9.7899 & 0.0052 & -1.30 & 0.950 & 0.222 & 12.17 & 0.57 \\ 1180 & 0.1480 & 1.1818 & 24.7152 & 0.0173 & -1.71 & 0.963 & -0.184 & 11.80 & 2.30 \\ 1280 & 0.0913 & 0.9437 & 34.4175 & 0.0158 & -1.86 & 0.975 & -0.632 & 9.43 & 3.66 \\ 1400 & 0.3023 & 1.3528 & 13.5434 & 0.0145 & -1.45 & 1.000 & 0.060 & 13.50 & 2.03\end{array}$


Sample 9: SB2A-54 108JD89B bio

$\mathrm{J}=0.0017465$

$\begin{array}{llllllllll}\mathrm{T} & 40(\mathrm{v}) & 40 / 39 & 37 / 39 & 36 / 39 & \mathrm{~K} / \mathrm{Ca} & \sum 39 \mathrm{Ar} & 40 \mathrm{Ar} * & \text { Age(Ma) } & \pm \\ 500 & 1.6792 & 2.8857 & 0.0555 & 0.1406 & 0.94 & 0.007 & 0.065 & 9.07 & 1.35 \\ 600 & 3.542 & 4.3366 & 0.0106 & 0.0756 & 1.66 & 0.031 & 0.163 & 13.61 & 0.42 \\ 700 & 2.8776 & 4.1938 & 0.0049 & 0.007 & 2.00 & 0.115 & 0.671 & 13.17 & 0.08 \\ 800 & 2.7441 & 4.1951 & 0.0055 & 0.0035 & 1.95 & 0.21 & 0.802 & 13.17 & 0.06 \\ 875 & 2.3364 & 4.1372 & 0.0053 & 0.0021 & 1.97 & 0.299 & 0.87 & 12.99 & 0.04 \\ 950 & 3.7583 & 4.164 & 0.0053 & 0.0019 & 1.96 & 0.444 & 0.883 & 13.07 & 0.03 \\ 1000 & 4.7329 & 4.1383 & 0.005 & 0.0018 & 1.99 & 0.627 & 0.884 & 12.99 & 0.03 \\ 1050 & 5.7376 & 4.1493 & 0.0079 & 0.0017 & 1.79 & 0.851 & 0.891 & 13.03 & 0.03 \\ 1100 & 2.9142 & 4.1382 & 0.0322 & 0.0014 & 1.18 & 0.967 & 0.909 & 12.99 & 0.04 \\ 1140 & 0.6492 & 4.1248 & 0.0421 & 0.0013 & 1.08 & 0.993 & 0.916 & 12.95 & 0.12 \\ 1250 & 0.1573 & 4.3416 & -0.0008 & -0.0004 & <-3 & 1 & 1.027 & 13.63 & 0.35\end{array}$

Total fusion age, $\mathrm{TFA}=13.03 \pm 0.02 \mathrm{Ma}$ (including $\mathrm{J}$ )

Weighted mean plateau age, WMPA $=13.03 \pm 0.02 \mathrm{Ma}$ (including $\mathrm{J}$ )

Inverse isochron age $=13.01 \pm 0.03 \mathrm{Ma} .(\mathrm{MSWD}=1.76 ; 40 \mathrm{Ar} / 36 \mathrm{Ar}=299.1 \pm 2.0)$

Steps used: $600,700,800,875,950,1000,1050,1100,1140,1250$, or $99 \%$ of $\sum 39 \mathrm{Ar}$.

Sample 10: cvi123 YR-6 hbl J=0.0055599

$\begin{array}{llllllllll}\mathrm{T} & 40(\mathrm{v}) & 40 / 39 & 37 / 39 & 36 / 39 & \mathrm{~K} / \mathrm{Ca} & \sum 39 \mathrm{Ar} & 40 \mathrm{Ar} * & \text { Age(Ma) } & \pm \\ 700 & 3.0039 & -2.1285 & 2.6980 & 0.5246 & -0.74 & 0.004 & -0.015 & -21.48 & 55.69 \\ 800 & 0.1617 & 1.7259 & 2.0146 & 0.0306 & -0.61 & 0.007 & 0.148 & 17.23 & 5.01 \\ 900 & 0.1902 & 1.5063 & 4.5539 & 0.0583 & -0.97 & 0.009 & 0.062 & 15.05 & 6.70 \\ 980 & 1.4182 & 1.3492 & 7.0393 & 0.0084 & -1.16 & 0.096 & 0.243 & 13.48 & 0.82 \\ 1020 & 2.9392 & 1.3782 & 7.1264 & 0.0052 & -1.16 & 0.346 & 0.346 & 13.77 & 0.53 \\ 1040 & 2.6835 & 1.3847 & 7.1416 & 0.0033 & -1.17 & 0.646 & 0.457 & 13.84 & 0.34 \\ 1060 & 1.2849 & 1.3971 & 7.1467 & 0.0029 & -1.17 & 0.799 & 0.492 & 13.96 & 0.35 \\ 1100 & 1.0047 & 1.3803 & 7.5509 & 0.0037 & -1.19 & 0.906 & 0.414 & 13.79 & 0.37 \\ 1200 & 0.6148 & 1.3832 & 10.3739 & 0.0049 & -1.33 & 0.967 & 0.280 & 13.82 & 0.40 \\ 1400 & 0.4178 & 1.3474 & 10.2699 & 0.0069 & -1.32 & 1.000 & 0.207 & 13.46 & 0.94\end{array}$

Sample 11: cvi126 88JD87 hbl J=0.0055365

$\begin{array}{llllllllll}\mathrm{T} & 40(\mathrm{v}) & 40 / 39 & 37 / 39 & 36 / 39 & \mathrm{~K} / \mathrm{Ca} & \sum 39 \mathrm{Ar} & 40 \mathrm{Ar}^{*} & \text { Age(Ma) } & \pm \\ 700 & 3.6776 & -2.2637 & 7.8450 & 0.8029 & -1.21 & 0.005 & -0.012 & -22.75 & 87.86 \\ 800 & 0.4390 & 0.4806 & 6.7759 & 0.1127 & -1.14 & 0.009 & -0.002 & 4.79 & 13.48 \\ 900 & 0.4169 & 0.5547 & 9.4970 & 0.1229 & -1.29 & 0.013 & -0.005 & 5.53 & 13.57 \\ 980 & 1.4128 & 1.1995 & 8.4105 & 0.0211 & -1.24 & 0.081 & 0.079 & 11.94 & 1.98 \\ 1020 & 2.0232 & 1.2829 & 8.5470 & 0.0110 & -1.24 & 0.252 & 0.157 & 12.77 & 0.98 \\ 1040 & 1.7745 & 1.2771 & 8.6253 & 0.0068 & -1.25 & 0.475 & 0.228 & 12.71 & 0.75 \\ 1060 & 1.7105 & 1.2989 & 8.6953 & 0.0051 & -1.25 & 0.737 & 0.286 & 12.93 & 0.51 \\ 1100 & 0.9283 & 1.2985 & 8.7807 & 0.0063 & -1.26 & 0.861 & 0.246 & 12.92 & 0.58 \\ 1200 & 0.8430 & 1.3840 & 8.7422 & 0.0059 & -1.25 & 0.974 & 0.284 & 13.77 & 0.60 \\ 1400 & 0.2894 & 1.6878 & 8.7961 & 0.0089 & -1.26 & 1.000 & 0.274 & 16.78 & 0.93\end{array}$


Sample 12: cvi101 77JD90 Hb J $=0.0056870$

$\begin{array}{llllllllll}\mathrm{T} & 40(\mathrm{v}) & 40 / 39 & 37 / 39 & 36 / 39 & \mathrm{~K} / \mathrm{Ca} & \sum 39 \mathrm{Ar} & 40 \mathrm{Ar} * & \text { Age(Ma) } & \pm \\ 700 & 2.7320 & -2.1359 & 4.3178 & 0.6794 & -0.95 & 0.004 & -0.012 & -22.05 & 67.07 \\ 800 & 0.1906 & 1.4650 & 3.5715 & 0.0713 & -0.86 & 0.006 & 0.053 & 14.97 & 7.49 \\ 900 & 0.1395 & 1.8080 & 4.2583 & 0.0447 & -0.94 & 0.009 & 0.100 & 18.45 & 5.31 \\ 980 & 1.1553 & 1.3024 & 6.5921 & 0.0125 & -1.13 & 0.081 & 0.175 & 13.31 & 1.21 \\ 1030 & 3.0107 & 1.3466 & 6.7247 & 0.0052 & -1.14 & 0.436 & 0.346 & 13.76 & 0.51 \\ 1080 & 3.0763 & 1.3770 & 6.7319 & 0.0034 & -1.14 & 0.900 & 0.457 & 14.07 & 0.32 \\ 1130 & 0.3035 & 1.4173 & 7.3965 & 0.0065 & -1.18 & 0.930 & 0.303 & 14.48 & 0.79 \\ 1200 & 0.1897 & 1.2654 & 8.9342 & 0.0094 & -1.26 & 0.946 & 0.166 & 12.94 & 1.71 \\ 1400 & 0.5887 & 1.4480 & 8.3851 & 0.0076 & -1.24 & 1.000 & 0.258 & 14.79 & 0.66\end{array}$

Sample 13: cvi108

$\begin{array}{lll}\mathrm{T} & 40(\mathrm{v}) & 40 / 39 \\ 700 & 4.5725 & 0.6406 \\ 800 & 0.2384 & 1.5343 \\ 900 & 0.3044 & 1.4060 \\ 980 & 0.9665 & 1.4630 \\ 1020 & 1.6024 & 1.4753 \\ 1060 & 3.0528 & 1.4680 \\ 1100 & 0.6899 & 1.4817 \\ 1200 & 0.5149 & 1.4803 \\ 1400 & 0.4055 & 1.3424\end{array}$

41JD89 Hb J $=0.0056440$

$\begin{array}{lllllll}37 / 39 & 36 / 39 & \text { K/Ca } & \sum 39 A r & 40 A r * & \text { Age(Ma) } & \pm \\ 2.8326 & 0.2886 & -0.76 & 0.017 & 0.005 & 6.51 & 25.70 \\ 1.7219 & 0.0112 & -0.55 & 0.034 & 0.299 & 15.55 & 1.51 \\ 2.1133 & 0.0238 & -0.64 & 0.046 & 0.150 & 14.26 & 3.26 \\ 7.6304 & 0.0115 & -1.19 & 0.119 & 0.202 & 14.83 & 1.21 \\ 8.1709 & 0.0058 & -1.22 & 0.324 & 0.326 & 14.96 & 0.54 \\ 8.1473 & 0.0044 & -1.22 & 0.791 & 0.386 & 14.88 & 0.45 \\ 7.9172 & 0.0043 & -1.21 & 0.896 & 0.400 & 15.02 & 0.45 \\ 10.0073 & 0.0056 & -1.31 & 0.968 & 0.292 & 15.01 & 0.65 \\ 10.6674 & 0.0121 & -1.34 & 1.000 & 0.121 & 13.62 & 1.79\end{array}$

Sample 14: cvil15 73JD90 wr $\quad J=0.0056118$

$\begin{array}{llllllllll}\mathrm{T} & 40(\mathrm{v}) & 40 / 39 & 37 / 39 & 36 / 39 & \mathrm{~K} / \mathrm{Ca} & \sum 39 \mathrm{Ar} & 40 \mathrm{Ar} & \text { Age(Ma) } & \pm \\ 600 & 1.1952 & 1.9088 & 4.4393 & 0.0108 & -0.96 & 0.100 & 0.327 & 19.22 & 1.01 \\ 650 & 2.1020 & 1.9812 & 6.0487 & 0.0300 & -1.09 & 0.181 & 0.145 & 19.95 & 2.89 \\ 700 & 1.5393 & 2.1484 & 7.3128 & 0.0175 & -1.18 & 0.273 & 0.232 & 21.62 & 1.37 \\ 760 & 1.3565 & 2.2238 & 4.9821 & 0.0060 & -1.01 & 0.423 & 0.507 & 22.37 & 0.54 \\ 820 & 1.2370 & 2.2324 & 4.0900 & 0.0050 & -0.92 & 0.569 & 0.562 & 22.46 & 0.53 \\ 890 & 0.8788 & 2.2025 & 4.9546 & 0.0061 & -1.01 & 0.666 & 0.500 & 22.16 & 0.72 \\ 1000 & 1.1977 & 2.1927 & 4.6668 & 0.0084 & -0.98 & 0.777 & 0.424 & 22.06 & 0.66 \\ 1140 & 1.5845 & 2.1416 & 7.6958 & 0.0090 & -1.20 & 0.929 & 0.366 & 21.55 & 0.83 \\ 1400 & 0.8574 & 2.1176 & 18.1182 & 0.0141 & -1.57 & 1.000 & 0.136 & 21.31 & 1.55\end{array}$

Sample 15: cvi104 140JD90 Bi $\quad \mathrm{J}=0.0056690$

\begin{tabular}{|c|c|c|c|c|c|c|c|c|c|}
\hline & $40(v)$ & $40 / 39$ & $37 / 39$ & $36 / 39$ & $\mathrm{~K} / \mathrm{Ca}$ & $\sum 39 \mathrm{Ar}$ & 40Ar* & Age(Ma) & \\
\hline 600 & 0.7920 & 1.7549 & 0.1360 & 0.0254 & 0.56 & 0.003 & 0.189 & & 2.38 \\
\hline 700 & 5.4211 & 1.7037 & 0.1032 & 0.0889 & 0.68 & 0.010 & 0.061 & 17.34 & 8.05 \\
\hline 800 & & & 0.0196 & & 1.40 & & 0.600 & & \\
\hline 380 & 5.4503 & 2.30 & & & 1.61 & 0.1 & 0.82 & & \\
\hline 00 & & 2.28 & 0.0077 & & $1 . ?$ & 0.2 & 0.86 & & \\
\hline$m$ & & & & & & & & & \\
\hline & & & & & 1. & 0.5 & 0.8 & & \\
\hline & & & 0.0 & & & & & & \\
\hline 151 & 6.6442 & 2.2729 & 0.0262 & 0.0004 & $1.2^{\circ}$ & 0.989 & 0.94 & 23.10 & \\
\hline & & & & & & & & & \\
\hline
\end{tabular}


B. ${ }^{40} \mathrm{Ar} /{ }^{39} \mathrm{Ar}$ Laser Heating Data for Sanidine:

\begin{tabular}{llllll} 
CVI-C-103 & 46JD90 Sanidine (Early ignimbrite) & \multicolumn{2}{l}{$\mathrm{J}=0.0056754$} \\
$* 40 / 39$ & \pm & $37 / 39\left(\times 10^{2}\right)$ & \%Rad & Age $(\mathrm{Ma})$ & \pm \\
2.798785 & .001251 & 1.667485 & 96.06 & 28.431 & .037 \\
2.813552 & .002581 & .7221835 & 98.55 & 28.580 & .044 \\
2.819728 & .001419 & 1.279734 & 97.86 & 28.642 & .038 \\
2.817241 & .001644 & .9155689 & 95.77 & 27.617 & .039 \\
2.817874 & .001814 & .5829086 & 98.09 & 28.624 & .040 \\
Mean/1 Stand. Error of Mean & & 28.5788 & .0383 \\
CVI-C-106 & 33JD90C Sanidine (White Tuff?) & $\mathrm{J}=.0056594$ \\
*40/39 & \pm & $37 / 39\left(\times 10^{2}\right)$ & \%Rad & Age(Ma) & \pm \\
2.434780 & .002161 & 2.965937 & 98.87 & 24.689 & .037 \\
2.436857 & .001233 & .9744206 & 99.58 & 24.710 & .033 \\
2.430956 & .002206 & 1.443132 & 98.39 & 24.651 & .038 \\
2.430892 & .002180 & 1.595030 & 97.70 & 24.650 & .037 \\
2.425494 & .001797 & 2.141401 & 98.54 & 24.596 & .035 \\
Mean/1 Stand. Error of Mean & & 24.6592 & .0195
\end{tabular}

Mean/1 Stand. Error of Mean

$24.6592 \quad .0195$

CVI-C-109 61JD89 Sanidine (Wassuk Group Ash) J=.0056426

$\begin{array}{llllll}* 40 / 39 & \pm & 37 / 39\left(\times 10^{2}\right) & \text { \%Rad } & \text { Age(Ma) } & \pm \\ 2.386241 & .002195 & 6.163442 & 97.94 & 24.129 & .037 \\ 2.387095 & .001867 & 10.27776 & 99.04 & 24.138 & .035 \\ 2.435351 & .001661 & 7.108073 & 98.42 & 24.622 & .035 \\ 2.364236 & .001666 & 7.000838 & 98.88 & 23.908 & .034 \\ 2.375289 & .001428 & 7.728727 & 98.46 & 24.019 & .033 \\ 2.338547 & .004130 & 4.663660 & 96.84 & 23.650 & .051 \\ \text { Mean/1 Stand. Error of Mean } & & 24.1630 & .1115 \\ \text { CVI-C-110 } & \text { 36JD90 Sanidine (Red Tuff) } & \mathrm{J}=.0056389 \\ \text { *40/39 } & \pm & 37 / 39\left(\times 10^{2}\right) & \text { \%Rad } & \text { Age(Ma) } & \pm \\ 2.444237 & .001565 & 2.708232 & 98.81 & 24.695 & .046 \\ 2.434080 & .002490 & 2.457468 & 99.16 & 24.594 & .050 \\ 2.435410 & .001437 & 2.307991 & 99.01 & 24.607 & .034 \\ 2.430069 & .002470 & 2.502527 & 98.84 & 24.553 & .039 \\ 2.431813 & .002188 & 2.970779 & 98.86 & 24.571 & .037 \\ \text { Mean/l Stand. Error of Mean } & & 24.604 & .0246\end{array}$

CVI-C-112 42JD90 Sanidine (blocks in fault breccia) $\mathrm{J}=.0056286$

$\begin{array}{llllll}* 40 / 39 & \pm & 37 / 39\left(\times 10^{2}\right) & \% \text { Rad } & \text { Age(Ma) } & \pm \\ 2.353031 & .001279 & 1.476854 & 98.76 & 23.737 & .032 \\ 2.342927 & .000890 & 1.452611 & 97.21 & 23.636 & .031 \\ 2.362043 & .001877 & 2.275070 & 98.79 & 23.827 & .035 \\ 2.345216 & .002247 & 1.410797 & 97.02 & 23.659 & .037 \\ 2.360654 & .001402 & 1.735940 & 98.64 & 23.813 & .033 \\ \text { Mean/1 Stand. Error of Mean } & & 23.7344 & .0388\end{array}$

C. K-Ar Data:

208JD88 Hornblende (Ash bed in Wassuk Group) (Inst. Human Origins/A. Deino)

${ }^{40} \mathrm{Ar}$ rad (moles/gm) $=7.481 \mathrm{E}-11 \pm 9.233 \mathrm{E}-13 ; \%$ Radiogenic $\mathrm{Ar}=16.0$;

$\mathrm{K}+(\mathrm{wt} . \%)=0.4525 \pm .02$; Sample wt. $=0.34755 ;$ Age $=9.51 \pm 1.20$ (1 s.dev. $)$ 